\title{
The complete ISO spectrum of NGC $6302^{\star}$
}

\author{
F. J. Molster ${ }^{1,2}$, T. L. Lim ${ }^{3}$, R. J. Sylvester ${ }^{4}$, L. B. F. M. Waters ${ }^{1,5}$, M. J. Barlow ${ }^{4}$, D. A. Beintema ${ }^{6}$, \\ M. Cohen ${ }^{7}$, P. $\operatorname{Cox}^{8}$, and B. Schmitt ${ }^{9}$ \\ 1 Astronomical Institute "Anton Pannekoek", University of Amsterdam, Kruislaan 403, 1098 SJ Amsterdam, \\ The Netherlands \\ 2 School of Materials Science and Engineering, Georgia Tech, Atlanta, GA 30332-0245, USA \\ 3 Space Science Department, Rutherford Appleton Laboratory, Chilton, Didcot OX11 0QX, UK \\ 4 Department of Physics and Astronomy, University College London, Gower Street, London WC1E 6BT, UK \\ ${ }^{5}$ Instituut voor Sterrenkunde, K.U. Leuven, Celestijnenlaan 200B, 3001 Heverlee, Belgium \\ ${ }^{6}$ SRON Space Research Laboratory, PO Box 800, 9700 AV Groningen, The Netherlands \\ 7 Radio Astronomy Laboratory, 601 Campbell Hall, University of California, Berkeley, CA 94720, USA \\ 8 Institut d'Astrophysique Spatiale, Bât. 121, Université de Paris XI, 91405 Orsay Cedex, France \\ 9 Laboratoire de Planetologie de Grenoble, Universite J. Fourier - CNRS, Bât. D de Physique, BP 53 , \\ 38041 Grenoble Cedex 9, France
}

Received 12 October 1999 / Accepted 22 March 2001

\begin{abstract}
We present the combined Infrared Space Observatory Short-Wavelength Spectrometer and LongWavelength Spectrometer 2.4-197 $\mu$ m spectrum of the Planetary Nebula NGC 6302 which contains in addition to strong atomic lines, a series of emission features due to solid state components. The broad wavelength coverage enables us to more accurately identify and determine the properties of both oxygen- and carbon-rich circumstellar dust. A simple model fit was made to determine the abundance and typical temperature of the amorphous silicates, enstatite and forsterite. Forsterite and enstatite do have roughly the same abundance and temperature. The origin and location of the dust in a toroidal disk around the central star are discussed.
\end{abstract}

Key words. circumstellar matter: planetary nebulae: individual: NGC 6302 - infrared: ISM: lines and bands

\section{Introduction}

The bipolar planetary nebula (PN) NGC 6302 is one of the brightest $\mathrm{PN}$ in our galaxy, and its central star is one of the hottest stars known (Ashley \& Hyland 1988; Pottasch et al. 1996). These properties suggest that the progenitor of NGC 6302 was massive, which is consistent with the high nebular abundance of nitrogen (Aller et al. 1981). The morphology of the nebula is strongly bipolar, with a dense, dusty torus (Lester \& Dinerstein 1984) and ionized polar regions as traced by the free-free radio emission (Gomez et al. 1993). Roche \& Aitken (1986) detected 8.6 and $11.3 \mu \mathrm{m}$ polycyclic aromatic hydrocarbon $(\mathrm{PAH})$ band emission from NGC 6302, usually associated with carbon-rich material, while the presence of oxygen-rich material was indicated by the detection of an $\mathrm{OH}$-maser

Send offprint requests to: F. J. Molster, e-mail: fjmolster@mvainc.com

* Based on observations with ISO, an ESA project with instruments funded by ESA Member States (especially the PI countries: France, Germany, The Netherlands and the UK) and with the participation of ISAS and NASA.
(Payne et al. 1988) and an $18 \mu \mathrm{m}$ amorphous silicate emission feature (Justtanont et al. 1992). NGC 6302 therefore can be considered as an important test of stellar evolution models: the nebular abundances provide constraints on the chemical evolution and nucleosynthesis processes, and the thermal emission from dust gives information on the mass loss and its geometry at the end of the AGB phase.

The infrared brightness and unique properties of NGC 6302 made it an excellent target for study with the Short Wavelength (SWS; de Graauw et al. 1996) and Long Wavelength (LWS; Clegg et al. 1996) Spectrometers on board the Infrared Space Observatory (ISO; Kessler et al. 1996). Several papers have already reported on some aspects of these ISO observations. One of the most remarkable results of the ISO observations of NGC 6302 was the discovery of strong emission due to crystalline silicates (Waters et al. 1996; Barlow 1998; Beintema 1998), as well as several other solid state emission bands, including those of water ice. A full inventory and characterization of these emission bands requires access to the entire 2.4-197 $\mu \mathrm{m}$ 
wavelength range that ISO offers. In this paper, we present for the first time the full grating spectrum of NGC 6302 . The present analysis of the spectrum will concentrate on the dust features.

This paper is organized as follows. In Sect. 2 we briefly describe the observations and data reduction. Section 3 discusses the placement of the continuum and presents the continuum subtracted spectrum. We identify most of the solid state bands. In Sect. 4 the results of a simple model to fit the spectral dust features and the continuum are presented and in Sect. 5 we discuss the results of our analysis.

\section{The observations and data reduction}

The SWS spectrum from 2.4 to $45 \mu \mathrm{m}$ of NGC 6302 was obtained with ISO on February 19, 1996 as an AOT01, with the highest resolution (speed 4 ). The total integration time was 6528 seconds. The spectra were reduced using the SWS offline processing software, version 7.0. For a description of flux and wavelength calibration procedures, see Schaeidt et al. (1996) and Valentijn et al. (1996). The main fringes in the $12.0-29.5 \mu \mathrm{m}$ part of the spectrum were removed using the Interactive (IA) data reduction package routine FRINGES. Major irregularities due to glitches and large drops or jumps in each detector were removed by hand. A comparison between the different detectors scanning the same wavelength region was used to determine the location of such jumps. The 12 SWS sub-spectra, when combined into a single spectrum, can show jumps in flux levels at band edges due to imperfect flux calibration or dark current subtraction. We have adjusted the different sub-bands, according to the expected source of discrepancy in each band, to form a continuous spectrum. Note that all the adjustments are well within the photometric absolute calibration uncertainties of $20 \%$ (Schaeidt et al. 1996). The relative error depends on band and flux but is typically much less than $5 \%$. The final resolving power $(\lambda / \Delta \lambda)$ is 1000 , apart from band $2 \mathrm{C}(7-12 \mu \mathrm{m})$ and $3 \mathrm{E}(27.5-29 \mu \mathrm{m})$ which have a resolving power of 500 and from band $1 \mathrm{~A}(2.4-2.6 \mu \mathrm{m})$ which was rebinned to a resolving power of 2000 .

The LWS spectrum from 40 to $197 \mu \mathrm{m}$ was obtained by combining seven full-range grating scan AOT L01 observations, obtained between 1996 August 31 and 1997 September 23, in revolutions 289, 482, 489, 503, 510, 671, and 678. An off-source spectrum, of a position 8 arcmin from NGC 6302, was also obtained in revolution 289. For a description of the flux and wavelength calibration procedures see Swinyard et al. (1996). Each of the seven observations consisted of six fast grating scans, with $0.5 \mathrm{~s}$ integration at each commanded grating position. The observations taken in revolutions 289 and 671 were sampled at $1 / 4$ of a spectral resolution element, the latter being $0.3 \mu \mathrm{m}$ in second order (detectors SW1-SW5 $\lambda \leq 93 \mu \mathrm{m}$ ) and $0.6 \mu \mathrm{m}$ in first order (detectors LW1LW5 $\lambda \geq 80 \mu \mathrm{m}$ ). All other observations were made with a sampling of $1 / 8$ of a resolution element. The total

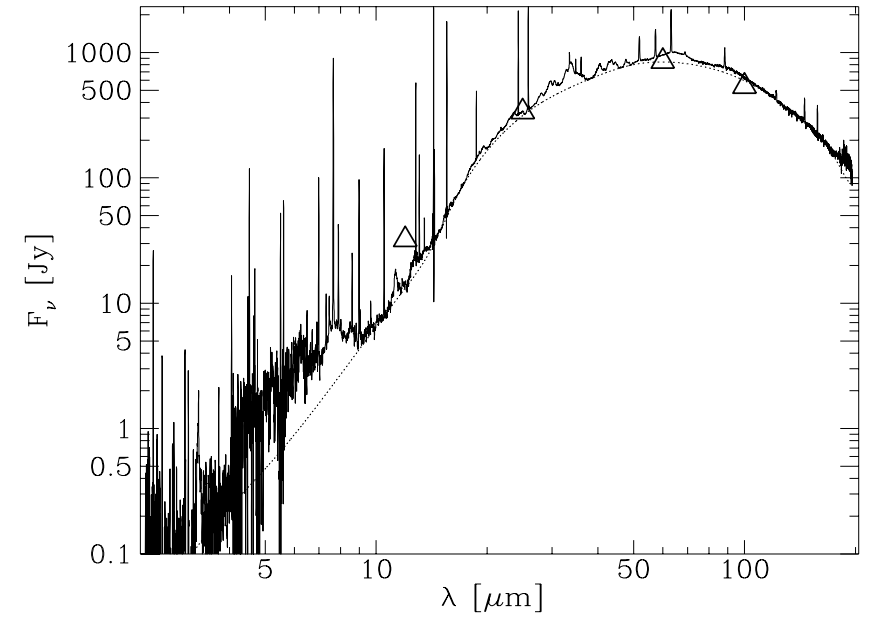

Fig. 1. The combined SWS and LWS spectrum of NGC 6302 (solid line). The IRAS broad-band flux densities are shown as triangles. Note that the IRAS $12 \mu \mathrm{m}$ band is severely influenced by a series of strong atomic lines. The dashed line is the spline continuum fit used for the continuum subtraction (see text and Fig. 2).

on-target integration time was $13243 \mathrm{~s}$. The data were reduced with the LWS offline processing software version 7.0. No scaling between the datasets was necessary before combining them to form a single dataset. The sub-spectra from the ten LWS detectors were individually rescaled to give the same flux in the regions of overlap, adopting a polynomial fit through all detectors to give scaling values. Each detector was scaled by a factor within $10 \%$ of unity. This is consistent with the formal absolute error of $10 \%$ per detector for a medium flux point source such as NGC 6302 (Swinyard et al. 1996). The scans were then averaged giving a relative error of no more than $3 \%$ per point. The ten sub-spectra were then merged to form a complete spectrum from 40 to $197 \mu \mathrm{m}$.

The whole SWS spectrum has to be multiplied by 1.22 to match the LWS-spectrum. The LWS observation was taken at the central position but for the SWS observation the satellite was pointed 5 arcsec away from the centre of NGC 6302. It should be noted that the sensitivity of the SWS decreases with distance from the centre. Another factor in the photometric differences between the two ISO spectrometers is the aperture size. The aperture of LWS is circular with a diameter of about 80 arcsec and the aperture size for SWS is rectangular and varies from band to band, from 14 arcsec $\times 20$ arcsec for the shortest wavelengths to $20 \operatorname{arcsec} \times 33$ arcsec for the longest wavelengths. The final spectrum is shown in Fig. 1, together with the IRAS broadband photometry points. The differences found between the IRAS fluxes and the ISO-fluxes convolved with the IRAS system response curves are well within the quoted IRAS, SWS and LWS flux uncertainties. The IRAS 12 micron flux, which seems to deviate, is actually consistent with our ISO observations when the forbidden emission lines are taken into account. The combined spectrum shows a wealth of atomic lines which are 
described in Beintema \& Pottasch (1999) and a series of broad emission bands due to solid state features, which will be described in this paper.

\section{The dust features}

To aid the identification of the spectral features, we have subtracted a spline curve "continuum" fit to the spectrum. The fit was made through selected points where no feature was believed to be present, with the only other two constraints being that the fit was smooth (both in $F_{\nu}$ as well as in $F_{\lambda}$ ) and contained as much continuum as possible. This approach will however reduce the strength of the features; it is also possible that very broad features have been treated as continuum. Note that a spline fit continuum has no physical meaning and is only used to enhance the visibility of the sharp solid state features. The continuum-subtracted spectrum is shown in Fig. 2. We find a wealth of solid state features. The dust features naturally divide the spectrum into a carbon-rich and an oxygen-rich part. Apart from the forbidden emission lines, the spectrum below $15 \mu \mathrm{m}$ is dominated by the aromatic infrared bands (AIBs) and above $15 \mu \mathrm{m}$ by O-rich dust features.

In Table 1 we list features found in the spectrum. The features were fitted with Gaussians using a "local" continuum, i.e. the flux apart from the feature(s), and which was not necessarily the same as the overall continuum. Since the errors are largely determined by this local continuum, we estimated our errors by fitting the data for different baseline continua. The spread in these results were used to calculate the errors, which are quoted in Table 1. This local fitting may prevent the discernment of some broad spectral features under narrow features. Although most features are fitted reasonably well with a Gaussian shape, we do not claim this to be the real shape. Since a lot of features are blended with others, this method is a systematical and reproducable way of determining the strength and position of the features. Features visible at $12.8,13.6,14.2,14.8,15.3,15.8,16.2$ and $25.8 \mu \mathrm{m}$ are not listed since they are possibly instrumental artifacts, caused by the presence of very strong forbidden emission lines on top of them (see below).

In the following sub-sections, we identify the different structures found in the continuum-subtracted spectrum. In case of doubt about the reality of features in the SWS wavelength range we compared the two scan directions and, if possible, the AOT06 spectra of NGC 6302 taken on 20 February and 9 March 1997, with the final spectrum. For the LWS range we checked for the presence of the feature in the individual observations, scans and subspectra.

\subsection{The 2.4-12 $\mu \mathrm{m}$ region; C-rich dust}

The continuum subtracted spectrum from 2.4 to $12 \mu \mathrm{m}$ (Fig. 2) is dominated by the well known AIBs at 3.3, 3.4, $6.2,7.7,8.5,11.0$ and $11.3 \mu \mathrm{m}$, usually attributed to PAHs
(Allamandola et al. 1989; Puget \& Leger 1989). Hence there is a clear indication of a C-rich dust environment, as first found by Roche \& Aitken (1986). A plateau is present at the long wavelength side of the $11.3 \mu \mathrm{m}$ feature extending to roughly $11.7 \mu \mathrm{m}$.

\subsection{The 12-17 $\mu \mathrm{m}$ region; an uncertain area of the spectrum}

The reality and strength of the features from 12 to $17 \mu \mathrm{m}$ is uncertain. We do see structures in our spectrum at 12.8 , 13.6, 14.2, 14.8, 15.3, 15.8, and $16.2 \mu \mathrm{m}$. In other sources $\mathrm{PAH}$ features are seen at 12.8, 13.6, 14.2 and $16.2 \mu \mathrm{m}$ (Hony et al. 2000; Van Kerckhoven et al. 2000). However this region in NGC 6302 is one with many very strong forbidden emission lines, some of which lie on top of the continuum features. The resultant shape of the continuum may be perturbed due to detector problems caused by shotnoise (Morris, private communication) and a comparison between the up and down scans does reveal that the two scan directions show a systematic offset with respect to each other at these wavelengths. We have also checked the SWS-AOT06 observation and found similar differences between the up and down scans, but also the absence of e.g. the $16.2 \mu \mathrm{m}$ feature. Further, there is also evidence for spurious features in the instrumental responsivity files at 13.6 and $14.2 \mu \mathrm{m}$ (Morris, private communication). Therefore although features are likely to be present in this wavelength region, the reality of the shape, strength and even presence of the features found in the $12-17 \mu \mathrm{m} \mathrm{spec}-$ trum of NGC 6302 remains uncertain, so they are not listed in Table 1.

\subsection{The 17-200 $\mu \mathrm{m}$ region; O-rich dust}

The spectral region between 15 and $50 \mu \mathrm{m}$ is characterized by a wealth of relatively sharp emission features. Most of the emission features can be identified with the Mg-rich crystalline silicates: forsterite $\left(\mathrm{Mg}_{2} \mathrm{SiO}_{4}\right)$ and enstatite $\left(\mathrm{MgSiO}_{3}\right)$. The identification of the crystalline silicate features is based on the laboratory measurements of Koike \& Shibai (1998) and Jäger et al. (1998), see Table 1. The wavelength position of the different features, especially at the long wavelengths, indicate that there is hardly any Fe present $(\mathrm{Mg} /(\mathrm{Fe}+\mathrm{Mg})>0.99)$ in the crystalline silicates. The different laboratory data sets for enstatite, which in general are in agreement about the position and strength of the features, do not agree in the details of their spectral shape. Therefore it is difficult to firmly identify some of these features to enstatite and in Table 1 they are listed as "possibly enstatite".

Amorphous silicates may well be present as indicated by the relatively broad emission feature at $18 \mu \mathrm{m}$ (not separately measured in Table 1), which is too broad to be just due to crystalline silicates. The presence of a $10 \mu \mathrm{m}$ silicate feature is suppressed by the low temperature of the (silicate) dust (see Sect. 4) and the C-rich dust 


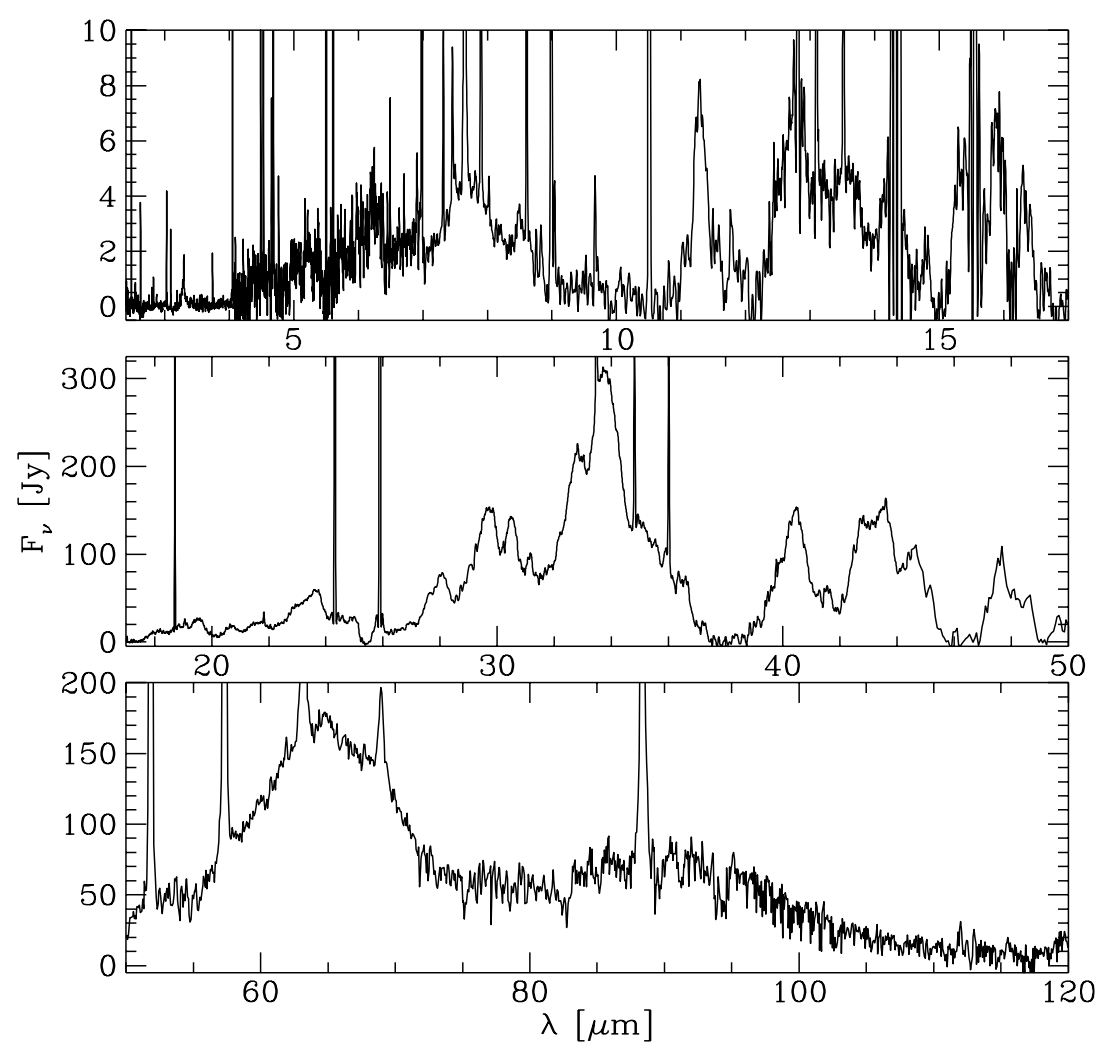

Fig. 2. The continuum-subtracted spectrum of NGC 6302 from 2.4 to $120 \mu \mathrm{m}$. The region from 2.4 to $17 \mu \mathrm{m}$ (top panel) is characterized by the presence of C-rich dust features. At longer wavelengths, the spectrum becomes dominated by O-rich dust with the crystalline silicates bands in the 17 to $50 \mu \mathrm{m}$ region (middle panel) and broad structures of crystalline $\mathrm{H}_{2} \mathrm{O}$ ice and probably hydrous silicates beyond $50 \mu \mathrm{m}$ (bottom panel).

continuum which is dominant at that wavelength. Another point which favours the presence of an amorphous dust component besides the crystalline dust, is the relative weakness of the crystalline dust features in the spectrum of NGC 6302 in this wavelength range compared with the laboratory measurements of pure crystalline silicates. Other sources with crystalline silicates also show evidence for amorphous silicates e.g. AFGL 4106 (Molster et al. $1999 \mathrm{~b})$. It is of course possible that other materials make a contribution to the 18 micron feature and this would give an alternative explanation for the suppression of the $10 \mu \mathrm{m}$ band, but we have found no reasonable candidate (yet).

The strength of the $20.7 \mu \mathrm{m}$ band is not completely reproduced by enstatite (see Sect. 4). This might be an indication of the presence of quartz $\left(\mathrm{SiO}_{2}\right)$, which shows a very prominent peak at this wavelength. Unfortunately, the other emission peaks of quartz are at much shorter wavelengths, and are suppressed due to the quartz being at low temperature compared to the hot C-rich dust. Therefore it is difficult to prove the presence of quartz based on this single feature. If quartz is present it will be only in small amounts.

The broad feature peaking at $63.6 \mu \mathrm{m}$, hereafter referred to as the $60 \mu \mathrm{m}$ feature, is probably a blend of diopside (Koike et al. 2000) and crystalline $\mathrm{H}_{2} \mathrm{O}$ ice with probably a small contribution from enstatite. This feature, as well as the $43-\mu \mathrm{m}$ ice feature, was observed by Omont et al. (1990) with KAO in the spectrum of IRAS $09371+1212$
(Frosty Leo), while Barlow (1998) reported the presence of $60 \mu \mathrm{m}$ emission in several dusty circumstellar shells and Sylvester et al. (1999) reported the ice emission in several $\mathrm{OH} / \mathrm{IR}$ stars. Laboratory data published by Bertie et al. (1969) and Schmitt et al. (1998) show that crystalline $\mathrm{H}_{2} \mathrm{O}$ ice has two strong bands, one at about $43 \mu \mathrm{m}$, the other at roughly $62 \mu \mathrm{m}$ with a weak shoulder at $52 \mu \mathrm{m}$. All these features are also present in the spectrum of NGC 6302 and especially the 43 and 60 micron feature cannot be explained by enstatite and/or diopside alone. Together with the expected presence of water-ice in a cold O-rich environment, the identification of crystalline water ice is very robust. However, it should be noted that a $52 \mu \mathrm{m}$ feature is also seen in $\eta$ Car, which has no further indication for crystalline $\mathrm{H}_{2} \mathrm{O}$ ice. Therefore we do not exclude that this feature is (a blend with) another dust species, probably a crystalline silicate.

There remain a handful of yet unidentified features, indicating the presence of other dust species. The broad feature at $91 \mu \mathrm{m}$ indicates that there must be a very cool dust component present. A feature around $100 \mu \mathrm{m}$ in HD 147527 was suggested by Malfait et al. (1999) to be due to the presence of a hydrous silicate. Hydrous silicates often show a broad peak in this wavelength range (Koike \& Shibai 1990) and should only exist at low temperatures. However, we do not know of a mechanism that would produce hydrous silicates in the surroundings of NGC 6302. Furthermore the peak in HD 147527 is quite shifted from the one in NGC 6302, which makes it 
Table 1. The characteristics of the dust features in the spectrum of NGC 6302. The quoted errors are derived by multiple independent fits to the different spectral features.

\begin{tabular}{|c|c|c|c|c|}
\hline $\begin{array}{c}\lambda \\
\mu \mathrm{m} \\
\end{array}$ & $\begin{array}{c}F W H M \\
\mu \mathrm{m} \\
\end{array}$ & $I_{\text {peak }} / I_{\text {cont }}$ & $\begin{array}{c}\text { Band flux } \\
10^{-13} \mathrm{Wm}^{-2}\end{array}$ & Identification \\
\hline $3.29(0.01)$ & $0.05(0.01)$ & $6.3(2.7)$ & $0.143(0.009)$ & PAH C-H stretch \\
\hline $3.41(0.01)$ & $0.05(0.02)$ & $2.1(0.7)$ & $0.027(0.006)$ & PAH C-H stretch \\
\hline $6.24(0.01)$ & $0.20(0.01)$ & $1.71(0.04)$ & $0.341(0.010)$ & PAH C-C stretch \\
\hline $7.66(0.05)$ & $0.69(0.02)$ & $1.74(0.08)$ & $0.87(0.26)$ & PAH C-C stretch \\
\hline $8.56(0.04)$ & $0.36(0.03)$ & $1.42(0.08)$ & $0.239(0.012)$ & PAH C-H bending in plane \\
\hline $11.01(0.03)$ & $0.13(0.07)$ & $1.180(0.029)$ & $0.05(0.04)$ & PAH+artifact? \\
\hline $11.29(0.01)$ & $0.27(0.07)$ & $1.77(0.07)$ & $0.49(0.11)$ & PAH C-H bending out of plane \\
\hline $18.10(0.02)$ & $0.75(0.13)$ & $1.125(0.018)$ & $0.96(0.17)$ & enstatite + amorphous silicate \\
\hline $18.97(0.01)$ & $0.68(0.06)$ & $1.128(0.006)$ & $1.05(0.07)$ & unidentified + amorphous silicate? \\
\hline $19.56(0.01)$ & $0.45(0.02)$ & $1.135(0.009)$ & $0.79(0.09)$ & forsterite \\
\hline $20.67(0.01)$ & $0.41(0.02)$ & $1.070(0.009)$ & $0.39(0.04)$ & possibly enstatite + quartz? \\
\hline $21.65(0.03)$ & $1.03(0.06)$ & $1.093(0.014)$ & $1.37(0.30)$ & possibly enstatite \\
\hline $23.09(0.10)$ & $1.31(0.51)$ & $1.189(0.012)$ & $3.7(1.7)$ & unidentified \\
\hline $23.71(0.02)$ & $0.58(0.09)$ & $1.157(0.028)$ & $1.4(0.5)$ & forsterite \\
\hline $27.52(0.07)$ & $0.38(0.12)$ & $1.070(0.032)$ & $0.47(0.26)$ & forsterite \\
\hline $28.01(0.04)$ & $0.66(0.17)$ & $1.139(0.032)$ & $1.5(0.4)$ & enstatite \\
\hline $29.08(0.04)$ & $0.66(0.09)$ & $1.079(0.011)$ & $0.91(0.17)$ & enstatite \\
\hline $29.74(0.03)$ & $0.66(0.05)$ & $1.199(0.025)$ & $2.2(0.4)$ & unidentified \\
\hline $30.51(0.01)$ & $0.44(0.04)$ & $1.165(0.014)$ & $1.23(0.12)$ & unidentified \\
\hline $31.11(0.02)$ & $0.24(0.03)$ & $1.050(0.004)$ & $0.209(0.28)$ & unidentified \\
\hline $32.73(0.02)$ & $0.94(0.12)$ & $1.236(0.018)$ & $3.6(0.5)$ & unidentified \\
\hline $33.79(0.01)$ & $0.98(0.04)$ & $1.441(0.030)$ & $6.8(0.5)$ & forsterite \\
\hline $39.86(0.06)$ & $1.25(0.14)$ & $1.092(0.009)$ & $1.49(0.05)$ & unidentified \\
\hline $40.49(0.05)$ & $0.80(0.03)$ & $1.176(0.014)$ & $1.82(0.20)$ & enstatite \\
\hline $41.56(0.06)$ & $0.92(0.21)$ & $1.085(0.013)$ & $1.00(0.34)$ & unidentified \\
\hline $42.73(0.09)$ & $0.89(0.14)$ & $1.152(0.035)$ & $1.71(0.7)$ & enstatite \\
\hline $43.54(0.06)$ & $0.83(0.21)$ & $1.179(0.023)$ & $1.8(0.6)$ & crystalline $\mathrm{H}_{2} \mathrm{O}$ \\
\hline $44.58(0.01)$ & $0.78(0.28)$ & $1.130(0.004)$ & $1.2(0.5)$ & unidentified \\
\hline $47.65(0.03)$ & $0.85(0.08)$ & $1.107(0.010)$ & $0.99(0.06)$ & unidentified \\
\hline $48.50(0.01)$ & $0.55(0.02)$ & $1.053(0.004)$ & $0.312(0.016)$ & unidentified \\
\hline $52.04(0.13)$ & $3.8(1.0)$ & $1.036(0.006)$ & $1.4(0.6)$ & crystalline $\mathrm{H}_{2} \mathrm{O} /$ silicate? \\
\hline $63.56(0.26)$ & $9.86(0.33)$ & $1.110(0.010)$ & $7.8(0.9)$ & crystalline $\mathrm{H}_{2} \mathrm{O}+$ diopside + enstatite \\
\hline $68.96(0.01)$ & $0.64(0.02)$ & $1.069(0.004)$ & $0.264(0.008)$ & forsterite \\
\hline $91.12(0.05)$ & $12.7(0.7)$ & $1.054(0.006)$ & $1.87(0.29)$ & unidentified \\
\hline
\end{tabular}

somewhat unlikely that this is the same material. As very little data for silicate minerals exists in this wavelength region, the identification remains open.

\section{Fitting the features and continuum}

Assuming that the dust shell is optically thin for wavelengths above $20 \mu \mathrm{m}$, we applied a very simple procedure to get an estimate for the relative amount of the crystalline and amorphous silicate dust fraction in the circumstellar dust shell around NGC 6302.

In order to determine the temperature and relative abundance of enstatite and forsterite we fitted continuum subtracted laboratory spectra to the continuum subtracted ISO spectrum of NGC 6302. Since one of the requirements of the spline fit to the continuum of NGC 6302 was that it should contain as much flux as possible, it also contains continuum flux caused by the crystalline silicate components. Although this is a small contribution, it is not negligible. So, we subtracted also a continuum of the laboratory spectra in the same way as we did for NGC 6302, i.e. by removing as much flux as possible but keeping the continuum smooth. Fitting the continuum subtracted spectrum has the advantage that one can immediately fit the temperature. It is also insensitive to the temperature of the continuum, which might be different from the temperature of the dust features.

The temperature of forsterite is mainly determined by the strength ratio of the features at 23.71 and $33.79 \mu \mathrm{m}$, while the temperature of enstatite depends on the ratio of the features around 28 and $40 \mu \mathrm{m}$. The laboratory data of Koike et al. (1999) were taken to fit the spectra. In this way we were able to determine independently the temperature of forsterite $(65 \pm 10 \mathrm{~K})$ and of enstatite $(70 \pm 10 \mathrm{~K})$, see Fig. 3. The main uncertainty in the derived temperature is caused by the determination of the continuum subtraction. Based on the laboratory mass absorption coefficients we were able to derive relative abundances of forsterite and enstatite. The derived enstatite 


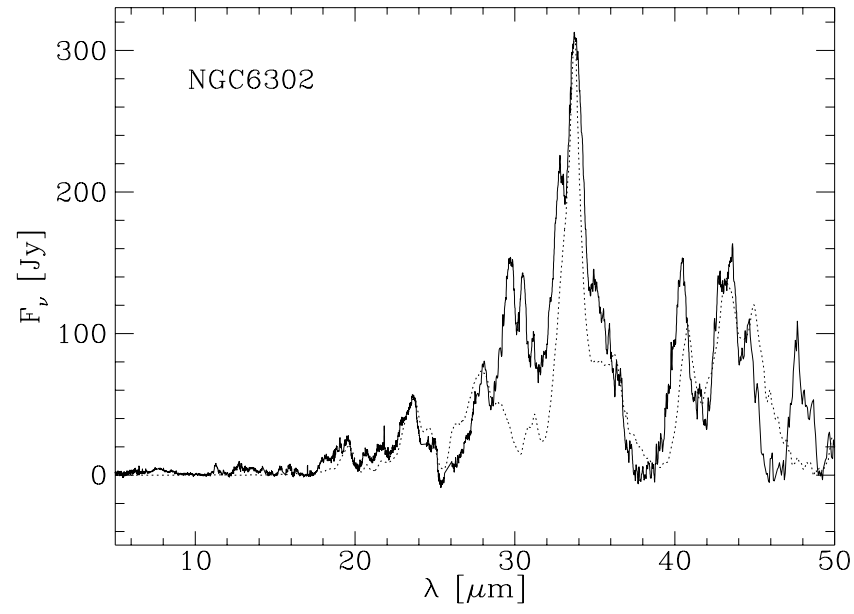

Fig. 3. A fit (dotted line) to the continuum subtracted spectrum (solid line) of NGC 6302. The mass absorption coeffcients of forsterite and the enstatite mix have been multiplied with Planck curves of 65 and $70 \mathrm{~K}$, respectively.

over forsterite mass ratio is 1.0, i.e. they are found in equal amounts. The typical error in the mass ratios is a factor 2 . This is mainly due to uncertainties in the temperature, which directly translate into differences in the mass fraction necessary to explain the strength of the features.

The temperature and relative abundance of the amorphous silicates have been determined by assuming that this material is mainly responsible for the infrared excess. We fitted a single temperature single grain $(0.1 \mu \mathrm{m})$ astronomical silicate, data from Ossenkopf et al. (1992), to the complete (not continuum subtracted) spectrum. Part of the continuum contains a small contribution of the crystalline silicate dust, the relative mass estimate of the amorphous silicate will be slightly (not more than a few percent) overestimated, but this is negligible compared to the other sources of uncertainty. The first major uncertainty is the fact that the infrared excess is too broad for a single temperature, single grain size approach. A fit to the spectrum up to $35 \mu \mathrm{m}$ results in a temperature of about $80 \mathrm{~K}$, but in a clear underestimation of the flux at longer wavelengths (see Fig. 4). A fit which would contain most of the flux results in a temperature of about $50 \mathrm{~K}$ (see Fig. 4). However, it is clear that this fit underestimates the flux at these wavelengths were the temperature and mass of the crystalline silicates has been determined $(<45 \mu \mathrm{m})$. It also is a much lower temperature than what has been derived for forsterite and seems therefore unlikely. Finally we also tried a fit with a similar temperature as found for forsterite (65 K, see Fig. 4). The amorphous silicate over forsterite mass ratios derived for these different temperatures are 6.3, 20.4 and 97 for respectively 80, 65 and $50 \mathrm{~K}$.

The gentle slope up to the $1.1 \mathrm{~mm}$ point (Hoare et al. $1992)$ indicates that large grains $(>10 \mu \mathrm{m})$ are present. They will be responsible for most of the flux at the long wavelengths, but due to their low temperature not for the flux below $40 \mu \mathrm{m}$. We therefore assume that the typical

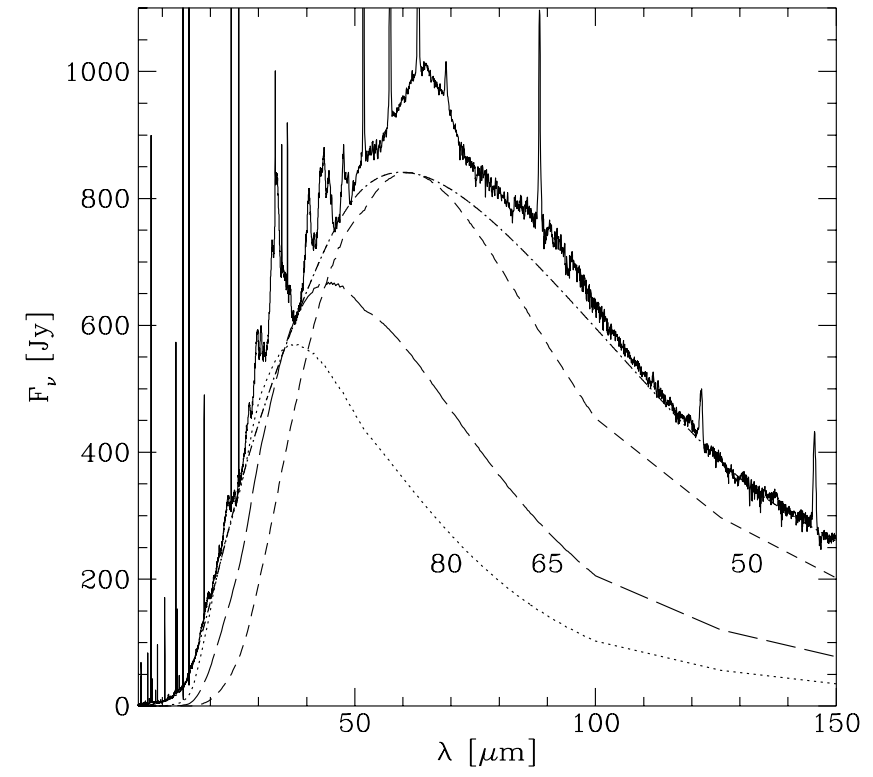

Fig. 4. The different fits to the spectrum of NGC 6302 (solid line). The dashed-dotted line is the continuum. The astronomical silicate is represented at 3 different temperatures at $80 \mathrm{~K}$ (dotted), $65 \mathrm{~K}$ (long-dashed) and $50 \mathrm{~K}$ (short-dashed). The kinks after $90 \mu \mathrm{m}$ are due to bad spectral coverage.

temperature of the small amorphous silicates, which are the source of flux below $40 \mu \mathrm{m}$, is somewhere between 65 and $80 \mathrm{~K}$ (see Fig. 4). We have calculated the dust mass necessary to explain the strength of the curves in Fig. 4 assuming a grainsize of $0.1 \mu \mathrm{m}$. We found that the fraction of crystalline silicates (enstatite and forsterite) in the total mass of small dust grains will be between 9 (when the small amorphous silicate dust grains are $65 \mathrm{~K}$ ) and $24 \%$ (when they are $80 \mathrm{~K}$ ). The composition and thus the abundance of the large grains is very difficult to determine, since they will not show spectral features.

We have made no effort to calculate the abundance of the water-ice or diopside. Both contribute significantly to the feature at $60 \mu \mathrm{m}$. Because of their blending it is very difficult to disentangle them and derive estimates for their temperature and thus their mass fraction.

As shown above the temperature for the amorphous silicates is difficult to determine, and the width of the infrared excess suggests a temperature distribution. The slope below $40 \mu \mathrm{m}$ suggests the presence of a population of relative warm (i.e. warmer than forsterite and enstatite) amorphous grains. We would like to note that in other studies, with full radiative transfer modeling (Molster et al. 1999b, 2001), it is shown that amorphous silicates are warmer than crystalline silicates. This may be due to a difference in chemical composition (i.e. the lack of Fe in the crystalline silicates), which results in different absorptivities in the UV, visual and near-IR wavelengths for the crystalline and amorphous silicates. 


\section{Discussion}

Both C-rich (PAH features) and O-rich (silicate features) chemistry is present in the spectrum of NGC 6302. A mixed chemistry is remarkable in an outflow, since one of the first formed stable molecules in an outflow is CO. The formation of CO is very efficient and completely "absorbs" the less abundant of the two. This leaves the most abundant atom to determine the dust composition. In the context of the massive N-rich type I planetary nebula NGC 6302, Justtanont et al. (1992) and Barlow (1993) pointed out that hot-bottom burning (HBB) at the base of the hydrogen envelopes of sufficiently massive AGB stars can eventually convert carbon dredged-up after a thermal pulse to nitrogen via the $\mathrm{CN}$ cycle. Thus the surface $\mathrm{C} / \mathrm{O}$ ratios and the grains condensed, can go from O-rich to C-rich and back again after each of the multiple thermal pulses experienced. This would allow both types of grains to be present in the resulting nebulae, without a single transition radius from O-rich to $\mathrm{C}$-rich material. This scenario would produce a mixed chemistry in the whole nebula.

It is difficult in this instance to explain the strength of the crystalline silicate features. They are much stronger than what is seen in normal outflow sources (Molster et al. 1999a). However, there are other evolved stars which show PAHs and crystalline silicates in their spectrum and are supposed to follow a different scenario. A famous example of this mixed chemistry is the Red Rectangle (Waters et al. 1998). This binary system formed a stable circumbinary disk while the star was still oxygen-rich. While the star evolved to a carbon-rich star, the disk remained. This is evidenced by the carbon-rich outflow and the oxygen-rich disk. The presence of a toroidal disk in NGC 6302 has long been known (Meaburn \& Walsh 1980; Lester \& Dinerstein 1984; Rodriguez et al. 1985), which, if stable, can explain the high degree of crystallinity by processes suggested by Molster et al. (1999a). The relatively high $\mathrm{mm}$ flux of NGC 6302 (Hoare et al. (1992) points to the presence of a population of large and cold grains. The presence of a stable disk naturally provides a suitable surrounding to produce large, and therefore cold, grains. This scenario would predict the O-rich material only to be present in the disk, with the C-rich dust dominating in the outflow and less pronounced in the disk.

In this context it is interesting to compare NGC 6302 with $\mathrm{CPD}-56^{\circ} 8032$. Although the central stars of these PNe are different, the spectra of the dust shells look quite similar. Both have roughly the same degree of crystallization, and the crystalline silicates have similar temperatures (Cohen et al. 1999). This is quite striking since the nebula around CPD-56 8032 is expected to be much younger $\left(\approx 10^{2}\right.$ yr, De Marco et al. 1997$)$ than the nebula around NGC $6302\left(\approx 10^{4} \mathrm{yr}\right.$, Terzian 1997). The expansion velocities are not that different, therefore it is likely that the crystalline material is not formed in the present day outflow, but more likely was already present around these objects, e.g. in a disk, before this outflow started. It has been suggested that we see here the destruction of Oortcloud like objects (Cohen et al. 1999), however the degree of crystallization is much higher than the crystallinity so far known for comets and interplanetary dust particles in our own solar system, which may make this Oort-cloud scenario unlikely.

The weakness of the PAH features with respect to the rest of the spectrum in NGC 6302 resembles the strength of the PAH features in the spectra of Luminous Blue Variables (LBVs) like R71 and AG Car, which show weak PAH emission and relatively strong crystalline silicates (Voors et al. 1999; Voors et al. 2000). Since these massive stars have a $\mathrm{C} / \mathrm{O}$ ratio lower than 1 , the formation of PAHs is not understood. It has been suggested that shocks might dissociate the $\mathrm{CO}$ and that the PAHs form in the after-shock region. If the PAHs in NGC 6302 are formed in a similar way, one would expect to find the PAHs in the neighbourhood of these shocked regions. Casassus et al. (2000) show that the $3.3 \mu \mathrm{m}$ emission is predominantly coming from the central region where the presentday outflow is expected to collide with the disk. Also the $\mathrm{C} / \mathrm{O}$ ratio of 0.88 (see Casassus et al. 2000) points to an oxygen-rich outflow. In the case of this scenario it is expected that the outflow and disk will be oxygen-rich and PAHs are only seen in the (after-)shocked regions. This would even allow the star to have been oxygen-rich for its entire life.

This last scenario explains the observed features best. However, one should keep in mind that a thorough understanding of the PAH formation in an oxygen dominated environment is still lacking. All three scenarios predict a different dust distribution. With the aid of future high spatial resolution observations in the PAH and crystalline silicate bands (and the continuum) we may be able to distinguish between the $\mathrm{HBB}$ scenario and the two different disk scenarios for NGC 6302.

Acknowledgements. FJM acknowledges support from NWO under grant 781-71-052 and under the talent fellowship program. LBFMW acknowledge financial support from an NWO "Pionier" grant. This work was partly supported by NWO Spinoza grant 08-0 to E. P. J. van den Heuvel. MC acknowledges the support of NASA under grant NAG 5-4884 and contract 961501 through JPL.

\section{References}

Allamandola, L. J., Tielens, A. G. G. M., \& Barker, J. R. 1989, ApJS, 71, 733

Aller, L. H., Ross, J. E., O'Mara, B. J., \& Keyes, C. D. 1981, MNRAS, 197, 95

Ashley, M. C. B., \& Hyland, A. R. 1988, ApJ, 331, 582

Barlow, M. J. 1993, Planetary Nebulae, IAU Symp. 155, ed. R. Weinberger, \& A. Ackerin (Dordrecht: Kluwer), 163

Barlow, M. J. 1998, A\&SS, 255, 315

Beintema, D. A. 1998, A\&SS, 255, 507

Beintema, D. A., \& Pottasch, S. R. 1999, A\&A, 347, 942 
Bertie, J. E., Labbé, H. J., \& Whalley, E. 1969, J. Chem. Phys., 50,4501

Casassus, S. P., Roche, P. F., \& Barlow, M. J. 2000, MNRAS, 314,657

Clegg, P. E., Ade, P. A. R., Armand, C., et al. 1996, A\&A, 315, L38

Cohen, M., Barlow, M. J., Sylvester, R. J., et al. 1999, ApJ, 513, L135

De Marco, O., Barlow, M. J., \& Storey, P. J. 1997, MNRAS, 292, 86

Gomez, Y., Rodríguez, L. F., \& Moran, J. M. 1993, ApJ, 416, 420

de Graauw, Th., Haser, L. N., Beintema, D. A., et al. 1996, A\&A, 315, L49

Hoare, M., Roche, P. F., \& Clegg, R. E. S. 1992, MNRAS, 258, 257

Hony, S., Van Kerckhoven, C., Peeters, E., et al. 2000, in proceedings of ISO beyond the peaks: The 2nd ISO workshop on analytical spectroscopy, ed. A. Salama, M. F. Kessler, K. Leech, \& B. Schulz, ESA-SP, 456, 63

Jäger, C., Molster, F. J., Dorschner, J., et al. 1998, A\&A, 339, 904

Justtanont, K., Barlow, M. J., Skinner, C. J., \& Tielens, A. G. G. M. 1992, ApJ, 392, L75

Kessler, M. F., Steinz, J. A., Anderegg, M. E., et al. 1996, A\&A, 315, L27

Koike, C., \& Shibai, H. 1990, MNRAS, 246, 332

Koike, C., Shibai, H. 1998, ISAS Report, No. 671

Koike, C., Tsuchiyama, A., Suto, H., et al. 1999, in Proceedings of the 32nd ISAS Lunar and Planetary Symposium, 32, 175

Koike, C., Tsuchiyama, A., Shibai, H., et al. 2000, A\&A, 363, 1115

Lester, D. F., \& Dinerstein, H. L. 1984, ApJ, 281, L67

Malfait, K., Walkens, C., Bowman, J., et al. 1999, A\&A, 345, 181

Meaburn, J., \& Walsh, J. R. 1980, MNRAS, 191, 5P

Molster, F. J., Yamamura, I., Waters, L. B. F. M., et al. 1999a, Nature, 401, 563
Molster, F. J., Waters, L. B. F. M., Trams, N., et al. 1999b, A\&A, 350, 163

Molster, F. J., Yamamura, I., Waters, L. B. F. M., et al. 2001, A\&A, 366, 923

Omont, A., Forveille, T., Moseley, S. H., et al. 1990, ApJ, 335, $270 \mathrm{~L}$

Ossenkopf, V., Henning, Th., \& Mathis, J. S. 1992, A\&A, 261, 567

Payne, H. E., Phillips, J. A., \& Terzian, Y. 1988, ApJ, 326, 368

Pottasch, S. R., Beintema, D., Dominguez-Rodriguez, F. J., et al. 1996, A\&A, 315, L261

Puget, J. L., \& Léger, A. 1989, ARA\&A, 27, 161

Roche, P. F., \& Aitken, D. K. 1986, MNRAS, 221, 63

Rodriguez, L. F., Garcia-Baretto, J. A., Canto, J., et al. 1985, MNRAS, 215, 353

Schaeidt, S. G., Morris, P. W., Salama, A., et al. 1996, A\&A, 315, L55

Schmitt, B., Quirico, E., Trotta, F., et al. 1998, Solar Syst. Ices Astrophys. Space Sci. Libr., 227, 199

Sylvester, R. J., Kemper, F., Barlow, M. J., et al. 1999, A\&A, 352,587

Swinyard, B. M., Clegg, P. E., Ade, P. A. R., et al. 1996, A\&A, 315, L43

Terzian, Y. 1997, ed. H. J. Habing, \& H. J. G. L. M. Lamers, Planetary Nebulae, Proc. IAU Symp., 180, 29

Valentijn, E. A., Feuchtgruber, H., Kester, D. J. M., et al. 1996, A\&A, 315, L60

Van Kerckhoven, C., Hony, S., Peeters, E., et al. 2000, A\&A, 357, 1013

Voors, R. H. M., Waters, L. B. F. M., Morris, P. W., et al. 1999, A\&A, 341, L67

Voors, R. H. M., Waters, L. B. F. M., de Koter, A., et al. 2000 , A\&A, 356, 501

Waters, L. B. F. M., Molster, F. J., de Jong, T., et al. 1996, A\&A, 315, L361

Waters, L. B. F. M., Waelkens, C., van Winckel, H., et al. 1998, Nature, 391, 868 